\title{
Simultaneous existence of amplified and attenuated Dyakonov surface waves
}

\author{
Tom G. Mackay ${ }^{1}$ \\ School of Mathematics and Maxwell Institute for Mathematical Sciences \\ University of Edinburgh, Edinburgh EH9 3FD, UK \\ and \\ NanoMM - Nanoengineered Metamaterials Group \\ Department of Engineering Science and Mechanics \\ Pennsylvania State University, University Park, PA 16802-6812, USA \\ Akhlesh Lakhtakia \\ NanoMM - Nanoengineered Metamaterials Group \\ Department of Engineering Science and Mechanics \\ Pennsylvania State University, University Park, PA 16802-6812, USA
}

\begin{abstract}
The propagation of Dyakonov surface waves guided by the planar interface of (i) an isotropic dielectric material and (ii) a homogenized uniaxial dielectric composite material comprising both passive and active component materials was theoretically investigated, under the assumption that the optic axis of the uniaxial partnering material lies wholly in the interface plane. For a certain range of the volume fraction of the active component material, the uniaxial partnering material is neither wholly dissipative nor wholly active and the Dyakonov surface waves propagating in certain directions amplify but the Dyakonov surface waves propagating in other directions attenuate.
\end{abstract}

\section{Introduction}

Major nanotechnological developments are currently spurring the conceptualization and occasional realization of metamaterials, which are engineered materials with exotic optical properties [1]. Notably, composite materials comprising both active and dissipative component materials seem to transcend merely the ability to overcome dissipation [2-5]. As an example, a random mixture of aligned spheroidal particles, both active and dissipative, may be theoretically equivalent to a uniaxial dielectric material in which a plane wave propagating in a particular direction can exhibit amplification but a second plane wave propagating in a different direction gets attenuated [6]. Furthermore, a multilaminate structure comprising alternate active and dissipative component layers may be theoretically equivalent to a birefringent material in which planewave polarization states can be controlled [7]. Homogenization formalisms also predict the possibilities of (i) an isotropic chiral material in which circularly polarized light of one handedness is amplified but circularly polarized light of the other handedness is attenuated [8], and (ii) a biaxial dielectric material which amplifies incident light of one linear polarization state but absorbs incident light of the orthogonal polarization state [9].

\footnotetext{
${ }^{1} \mathrm{E}-$ mail: T.Mackay@ed.ac.uk.
} 
In this short paper, we focus on Dyakonov surface waves [10-12] guided by the planar interface of an isotropic dielectric material and an anisotropic dielectric composite material comprising both active and dissipative component materials. Previous theoretical as well as experimental investigations have shown that, if both partnering materials are nondissipative (and inactive), Dyakonov-surface-wave propagation is generally possible only for propagation directions in very small angular sectors [13,14]. Nevertheless, Dyakonov surface waves are attractive as they offer considerable potential for long-range optical communications $[15,16]$. Furthermore, the range of propagation directions may be greatly increased if the anisotropic partnering material is either a hyperbolic material $[17,18]$ or a periodically nonhomogeneous material [19], or if at least one of the two partnering materials is dissipative [20].

We report here on the prospects for amplification and attenuation of Dyakonov surface waves, by solving the corresponding canonical boundary-value problem [22] in which an inactive isotropic material occupies the half-space $z<0$ while an anisotropic engineered material, comprising both active and dissipative component materials, occupies the half-space $z>0$. The unit vectors parallel to the Cartesian axes are written as $\underline{\hat{u}}_{x}$, $\underline{\hat{u}}_{y}$, and $\underline{\hat{u}}_{z}$. The free-space wave number is written as $k_{0}=\omega \sqrt{\varepsilon_{0} \mu_{0}}$ with $\omega$ being the angular frequency and $\varepsilon_{0}$ and $\mu_{0}$ being the permittivity and permeability of free space, respectively.

\section{Theoretical Preliminaries}

Both Marchevskii et al. [10] and Dyakonov [11] have provided the formalism for surface-wave propagation guided by the planar interface of an isotropic dielectric material and a uniaxial dielectric material whose optic axis is oriented to lie wholly in the interface plane. We are content to provide only essential details here, while referring the interested reader to more recent literature for greater illumination $[13,21,22]$.

The uniaxial partnering material, labeled $\mathcal{A}$, is taken to occupy the half-space $z>0$. Its relative permittivity dyadic is expressed as

$$
\underline{\varepsilon}_{\mathcal{A}}=\varepsilon_{\mathcal{A}}^{\mathrm{s}} \underline{\underline{I}}+\left(\varepsilon_{\mathcal{A}}^{\mathrm{t}}-\varepsilon_{\mathcal{A}}^{\mathrm{s}}\right) \underline{\hat{u}} \underline{\hat{u}}
$$

with $\underline{\underline{I}}$ being the identity dyadic [23] and the optic axis being parallel to the unit vector

$$
\underline{\hat{u}}=\underline{\hat{u}}_{x} \cos \psi+\underline{\hat{\imath}}_{y} \sin \psi
$$

lying wholly in the $x y$ plane. The eigenvalue $\varepsilon_{\mathcal{A}}^{\mathrm{s}}$ governs the propagation of ordinary plane waves, while the eigenvalues $\varepsilon_{\mathcal{A}}^{\mathrm{t}}$ and $\varepsilon_{\mathcal{A}}^{\mathrm{s}}$ jointly govern the propagation of extraordinary plane waves, in material $\mathcal{A}$ [23, 24]. The half-space $z<0$ is occupied by an isotropic dielectric material, labeled $\mathcal{B}$, whose relative permittivity is denoted by $\varepsilon_{\mathcal{B}}$.

Without loss of generality, the Dyakonov surface wave is considered to propagate parallel to $\underline{\hat{u}}_{x}$ in the $x y$ plane with $k_{0} q$ denoting the surface wavenumber. Thus, the direction of propagation is oriented at angle $-\psi$ with respect to the optic axis. The normalized propagation constant $q, \operatorname{Re}\{q\}>0$, is determined by solving the dispersion equation [11]

$$
\begin{aligned}
& \varepsilon_{\mathcal{A}}^{s}\left(\varepsilon_{\mathcal{B}} \alpha_{\mathcal{A} 1}+\varepsilon_{\mathcal{A}}^{s} \alpha_{\mathcal{B}}\right)\left(\alpha_{\mathcal{B}}+\alpha_{\mathcal{A} 2}\right) \tan ^{2} \psi \\
& \quad=\alpha_{\mathcal{A} 1}\left(\alpha_{\mathcal{B}}+\alpha_{\mathcal{A} 1}\right)\left(\varepsilon_{\mathcal{A}}^{s} \alpha_{\mathcal{B}} \alpha_{\mathcal{A} 2}+\varepsilon_{\mathcal{B}} \alpha_{\mathcal{A} 1}^{2}\right)
\end{aligned}
$$

where

$$
\left.\begin{array}{l}
\alpha_{\mathcal{A} 1}=\sqrt{q^{2}-\varepsilon_{\mathcal{A}}^{s}} \\
\alpha_{\mathcal{A} 2}=\sqrt{\varepsilon_{\mathcal{A}}^{t}\left[q^{2}\left(\frac{\cos ^{2} \psi}{\varepsilon_{\mathcal{A}}^{s}}+\frac{\sin ^{2} \psi}{\varepsilon_{\mathcal{A}}^{t}}\right)-1\right]}
\end{array}\right\}
$$

and

$$
\alpha_{\mathcal{B}}=\sqrt{q^{2}-\varepsilon_{\mathcal{B}}}
$$

are such that $\operatorname{Re}\left\{\alpha_{\mathcal{A} 1}\right\}>0, \operatorname{Re}\left\{\alpha_{\mathcal{A} 2}\right\}>0$, and $\operatorname{Re}\left\{\alpha_{\mathcal{B}}\right\}>0$. The Dyakonov surface wave grows in magnitude as it propagates if $\operatorname{Im}\{q\}<0$ but diminishes if $\operatorname{Im}\{q\}>0$. 
According to Eq. (3), if a Dyakonov surface wave can propagate for $\psi=\psi^{\star}$, then Dyakonov-surface-wave propagation is also possible for $\psi=-\psi^{\star}$ and $\psi=\pi \pm \psi^{\star}$. Parenthetically, these Dyakonov-surface-wave symmetries do not hold if the isotropic dielectric material occupying the half-space $z<0$ is endowed with a surface admittance [25].

\section{$3 \quad$ Numerical studies}

\subsection{Partnering materials}

In order to investigate the combined effects of dissipation and amplification in the partnering materials on Dyakonov-surface-wave propagation, material $\mathcal{A}$ was taken to be an engineered composite material comprising isotropic dielectric component materials $\mathcal{A} a$ and $\mathcal{A} b$. Their relative permittivities are denoted by $\varepsilon_{\mathcal{A} a}$ and $\varepsilon_{\mathcal{A} b}$ and their volume fractions by $f_{\mathcal{A} a}$ and $f_{\mathcal{A} b}=1-f_{\mathcal{A} a}$, respectively. The degree of anisotropy may be maximized by assuming that the component materials are randomly distributed as aciculate particles oriented parallel to $\underline{\hat{u}}[26]$. The Bruggeman homogenization formalism then provides the following estimates for the relative permittivity parameters of material $\mathcal{A}[27]$ :

$$
\left.\begin{array}{rl}
\varepsilon_{\mathcal{A}}^{\mathrm{s}}= & \frac{1}{2}\left[\left(f_{\mathcal{A} b}-f_{\mathcal{A} a}\right)\left(\varepsilon_{\mathcal{A} b}-\varepsilon_{\mathcal{A} a}\right)\right. \\
& \left.+\sqrt{\left[\left(f_{\mathcal{A} b}-f_{\mathcal{A} a}\right)\left(\varepsilon_{\mathcal{A} b}-\varepsilon_{\mathcal{A} a}\right)\right]^{2}+4 \varepsilon_{\mathcal{A} a} \varepsilon_{\mathcal{A} b}}\right] \\
\varepsilon_{\mathcal{A}}^{\mathrm{t}}= & f_{\mathcal{A} a} \varepsilon_{\mathcal{A} a}+f_{\mathcal{A} b} \varepsilon_{\mathcal{A} b}
\end{array}\right\} .
$$

In a similar vein, a laminated composite material could yield the desired high degree of anisotropy for material $\mathcal{A}[24]$.

For component material $\mathcal{A} a$ we chose a dissipative material with relative permittivity $\varepsilon_{\mathcal{A} a}=3+0.05 i$. For component material $\mathcal{A} b$ we chose an active material with relative permittivity $\varepsilon_{\mathcal{A} b}=2-0.03 i$. This value of $\varepsilon_{\mathcal{A} b}$ sits within the range typically employed for active components of metamaterials in the visible regime. As a specific illustration, a mixture of Rhodamine 800 and Rhodamine $6 \mathrm{G}$ exhibits a relative permittivity with imaginary part in the range $(-0.15,-0.02)$ and real part in the range $(1.8,2.3)$ across the frequency range 440-500 THz, depending upon the relative concentrations and the external pumping rate [5].

The Bruggeman estimates of $\varepsilon_{\mathcal{A}}^{\mathrm{s}}$ and $\varepsilon_{\mathcal{A}}^{\mathrm{t}}$ are plotted versus volume fraction $f_{\mathcal{A} b}$ in Fig. 1. Both $\operatorname{Im}\left\{\varepsilon_{\mathcal{A}}^{\mathrm{s}}\right\}>0$ and $\operatorname{Im}\left\{\varepsilon_{\mathcal{A}}^{\mathrm{t}}\right\}>0$ for $f_{\mathcal{A} b}<0.52$, so that the composite material $\mathcal{A}$ is dissipative. In contrast, material $\mathcal{A}$ is

active for $f_{\mathcal{A} b}>0.63$ since both $\operatorname{Im}\left\{\varepsilon_{\mathcal{A}}^{\mathrm{s}}\right\}<0$ and $\operatorname{Im}\left\{\varepsilon_{\mathcal{A}}^{\mathrm{t}}\right\}<0$ then. Most interestingly, for $f_{\mathcal{A} b} \in(0.52,0.63)$ material $\mathcal{A}$ is neither wholly active nor wholly dissipative since $\operatorname{Im}\left\{\varepsilon_{\mathcal{A}}^{\mathrm{s}}\right\}<0$ but $\operatorname{Im}\left\{\varepsilon_{\mathcal{A}}^{\mathrm{t}}\right\}>0$ then. In the next section, Dyakonov-surface-wave propagation is investigated for $f_{\mathcal{A} b}=0.4,0.58$, and 0.8, material $\mathcal{A}$ then being wholly dissipative, neither wholly active nor wholly dissipative, and wholly active, respectively.

The following values of the relative permittivity of material $\mathcal{B}$ were chosen for numerical investigation: $\varepsilon_{\mathcal{B}}=2.5,2.5+0.01 i$, and $2.5+0.2 i$.

\subsection{Surface-wave analysis}

Let us begin with the case in which material $\mathcal{B}$ is nondissipative: $\varepsilon_{\mathcal{B}}=2.5$. The real and imaginary parts of the normalized propagation constant $q$, as delivered from Eq. (3), are plotted against the angle $\psi$ for $f_{\mathcal{A} b} \in\{0.4,0.58,0.8\}$ in Fig. 2. Dyakonov-surface-wave propagation is possible for $\psi \in\left(55^{\circ}, 90^{\circ}\right)$ when $f_{\mathcal{A} 2}=0.4$, but for $\psi \in\left(0^{\circ}, 90^{\circ}\right)$ when $f_{\mathcal{A} b} \in\{0.58,0.8\}$. From the plots of $\operatorname{Im}\{q\}$, we infer that the Dyakonov surface wave is attenuated when $f_{\mathcal{A} b}=0.4$ but amplified when $f_{\mathcal{A} b} \in\{0.58,0.8\}$, regardless of direction of propagation (as given by $\psi$ ).

Parenthetically, the large angular existence domains (i.e., ranges of values $\psi$ for which Dyakonov-surfacewave propagation is possible) in Fig. 2 are attributable to the dissipative and active nature of material $\mathcal{A}$. Indeed, previous studies have revealed that if the partnering materials are characterized by relative permittivity parameters with nonzero imaginary parts then large angular existence domains can be obtained 

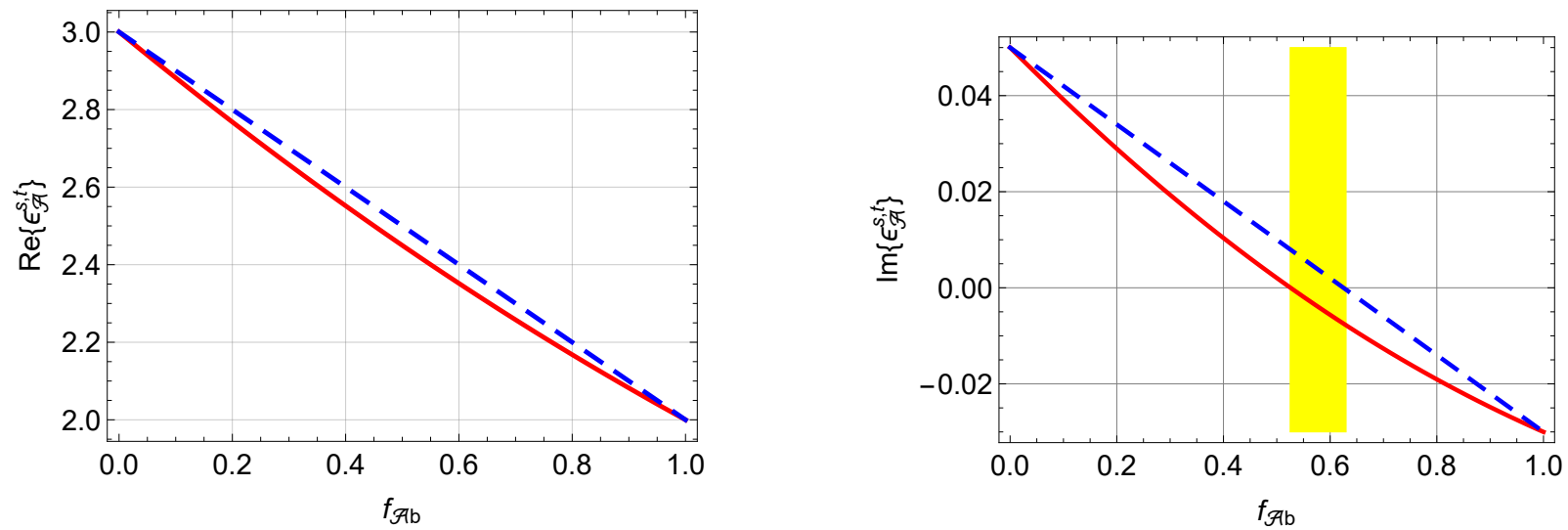

Figure 1: Real and imaginary parts of $\varepsilon_{\mathcal{A}}^{\mathrm{s}}$ (red solid curves) and $\varepsilon_{\mathcal{A}}^{\mathrm{t}}$ (blue dashed curves) plotted against volume fraction $f_{\mathcal{A} b}$. The range $f_{\mathcal{A} b} \in(0.52,0.63)$ for which $\operatorname{Im}\left\{\varepsilon_{\mathcal{A}}^{\mathrm{s}}\right\}$ and $\operatorname{Im}\left\{\varepsilon_{\mathcal{A}}^{\mathrm{t}}\right\}$ have opposite signs is indicated in yellow.
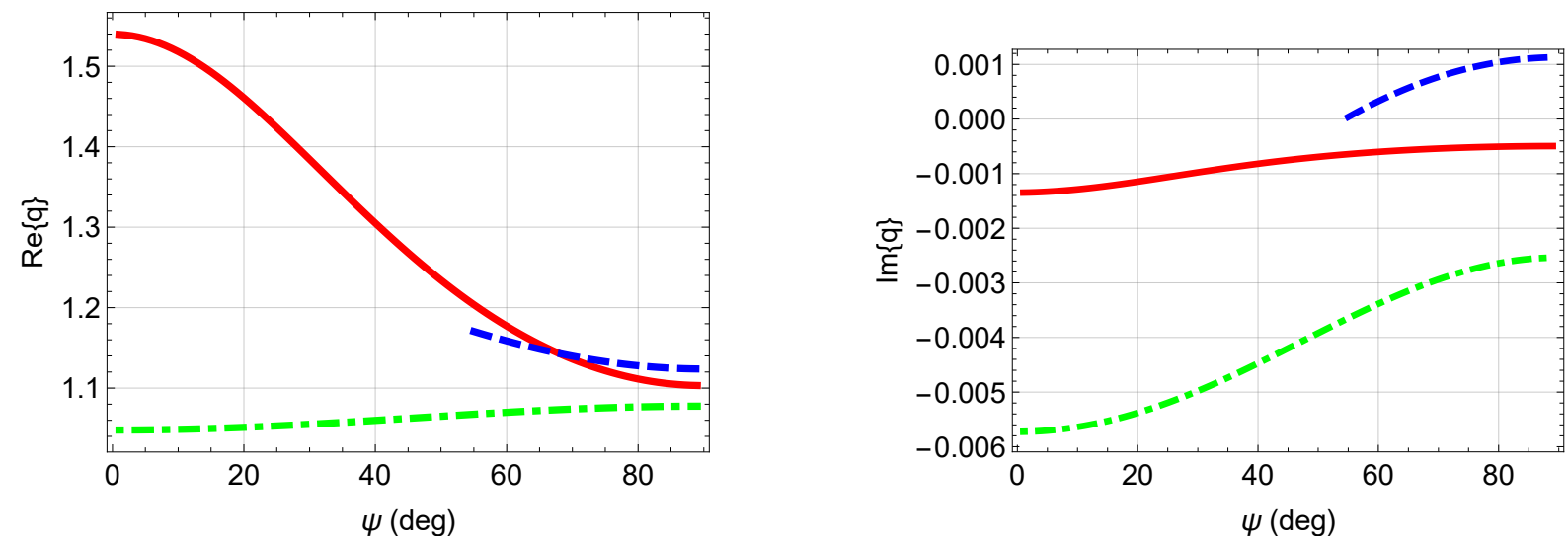

Figure 2: $\operatorname{Re}\{q\}$ and $\operatorname{Im}\{q\}$ plotted against $\psi \in\left[0^{\circ}, 90^{\circ}\right]$ for $f_{\mathcal{A} b}=0.4$ (blue dashed curves), 0.58 (red solid curves), and 0.8 (green broken dashed curves), when $\varepsilon_{\mathcal{B}}=2.5$.

for Dyakonov surface waves [20]. In contrast, when both partnering materials are taken to be nondissipative and nonactive, the angular existence domains are minuscule $[13,14]$.

Next we turn to the case in which material $\mathcal{B}$ is weakly dissipative: $\varepsilon_{\mathcal{B}}=2.5+0.01 i$. Plots of the real and imaginary parts of $q$ versus $\psi$ are provided in Fig. 3 for $f_{\mathcal{A} b} \in\{0.4,0.58,0.8\}$. The Dyakonov surface wave exists only for $\psi \in\left(63.9^{\circ}, 64.6^{\circ}\right)$ when $f_{\mathcal{A} b}=0.4$, but for $\psi \in\left(0^{\circ}, 90^{\circ}\right)$ when $f_{\mathcal{A} b} \in\{0.58,0.8\}$. The tiny angular existence domain for $f_{\mathcal{A} b}=0.4$ is typical for Dyakonov surface waves when both partnering materials are nondissipative and inactive $[13,14]$. From the plots of $\operatorname{Im}\{q\}$ we infer that the Dyakonov surface wave is attenuated when $f_{\mathcal{A} b}=0.4$ and amplified when $f_{\mathcal{A} b}=0.8$, regardless of the direction of propagation. But the case $f_{\mathcal{A} b}=0.58$ combines both characteristics since $\operatorname{Im}\{q\}<0$ for $\psi \in\left(0^{\circ}, 43^{\circ}\right)$ but $\operatorname{Im}\{q\}>0$ for $\psi \in\left(43^{\circ}, 90^{\circ}\right)$. Thus, the Dyakonov surface wave is attenuated for large values of $\psi$ but amplified for small values of $\psi$.

Lastly we consider the case of $\mathcal{B}$ being strongly dissipative: $\varepsilon_{\mathcal{B}}=2.5+0.2 i$. The real and imaginary parts of $q$ are plotted versus $\psi$ in Fig. 4 for $f_{\mathcal{A} b} \in\{0.4,0.58,0.8\}$. Dyakonov-surface-wave propagation is possible 

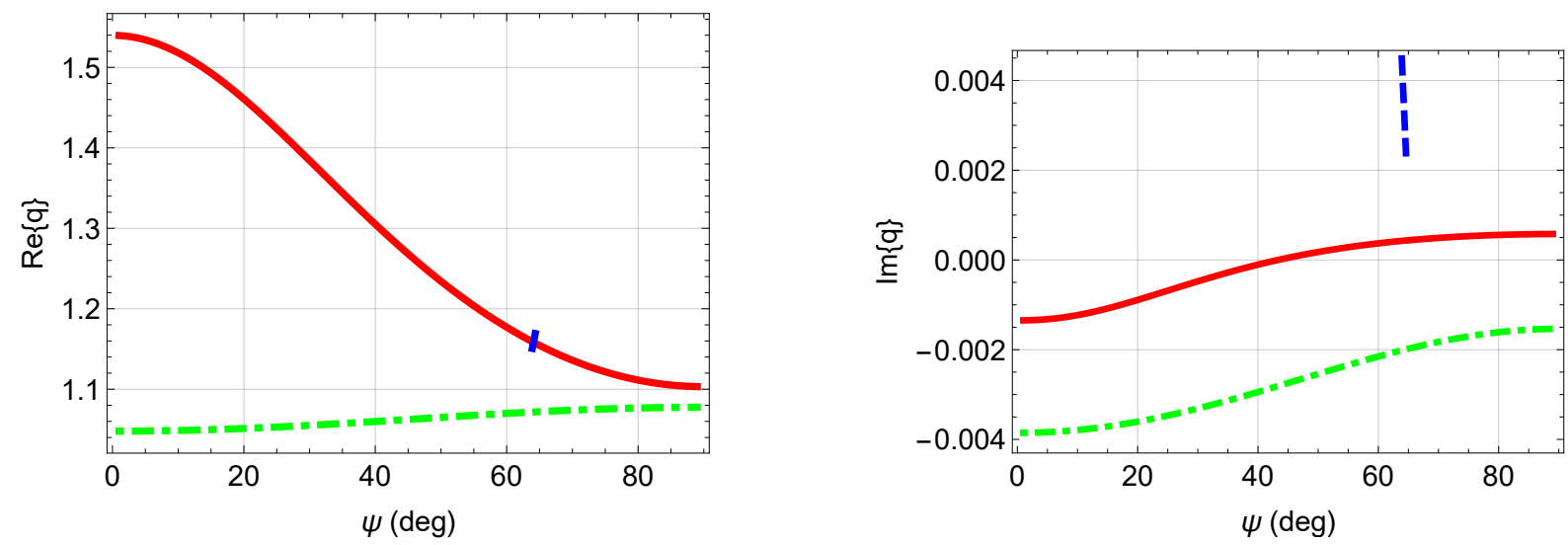

Figure 3: As Fig. 2 except that $\varepsilon_{\mathcal{B}}=2.5+0.01 i$.
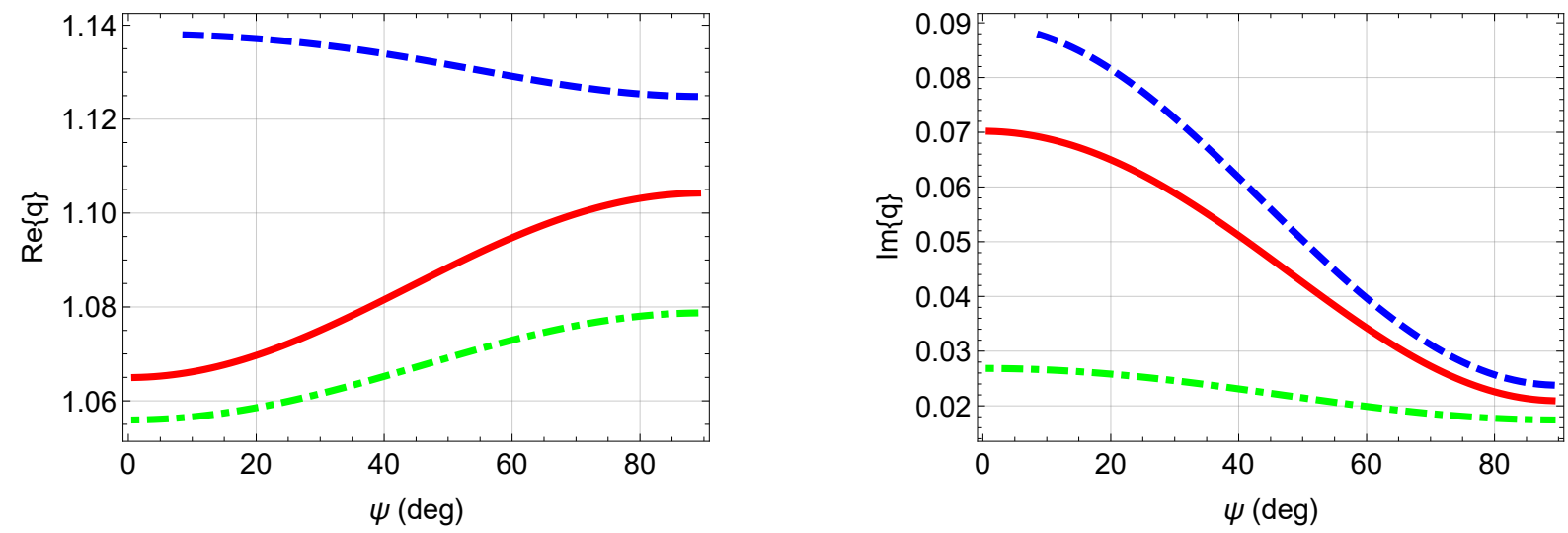

Figure 4: As Fig. 2 except that $\varepsilon_{\mathcal{B}}=2.5+0.2 i$.

for $\psi \in\left(9^{\circ}, 90^{\circ}\right)$ when $f_{\mathcal{A} b}=0.4$, but for all directions when $f_{\mathcal{A} b} \in\{0.58,0.8\}$. Furthermore, the Dyakonov surface wave is attenuated for all values of $f_{\mathcal{A} b}$ considered, regardless of direction of propagation.

\section{Closing remarks}

Dyakonov surface waves guided by the interface of nondissipative (and inactive) materials are generally restricted to tiny angular existence domains [13]. By allowing at least one of the partnering materials to be dissipative (or active), angular existence domains can be increased substantially [20]. The propagation of Dyakonov surface waves guided by the planar interface of an isotropic dielectric material and a homogenized uniaxial dielectric composite material comprising both passive and active component materials was theoretically investigated, with the optic axis of the uniaxial partnering material assumed to lie wholly in the interface plane. For a certain range of the volume fraction of the active component material, the uniaxial partnering material is neither wholly dissipative nor wholly active, Dyakonov-surface-wave propagation can occur in 
every direction in the interface plane, and the Dyakonov surface waves propagating in certain directions amplify but the Dyakonov surface waves propagating in other directions attenuate. These characteristics may be exploited for applications involving optical sensing and/or optical communications $[15,16]$.

Acknowledgments. AL is grateful to the Charles Godfrey Binder Endowment at Penn State for ongoing support of his research.

\section{References}

[1] R. M. Walser, Electromagnetic metamaterials, Proc. SPIE 4467 (2001) 1.

[2] S. Wuestner, A. Pusch, K. L. Tsakmakidis, J. M. Hamm, O. Hess, Overcoming losses with gain in a negative refractive index metamaterial, Phys. Rev. Lett. 105 (2010) 127401.

[3] Z.-G. Dong, H. Liu, T. Li, Z.-H. Zhu, S.-M. Wang, J.-X. Cao, S.-N. Zhu, X. Zhang, Optical loss compensation in a bulk left-handed metamaterial by the gain in quantum dots,' Appl. Phys. Lett. 96 (2010) 044104 .

[4] G. Strangi, A. De Luca, S. Ravaine, M. Ferrie, R. Bartolino, Gain induced optical transparency in metamaterials, Appl. Phys. Lett. 98 (2011) 251912.

[5] L. Sun, X. Yang, J. Gao, Loss-compensated broadband epsilon-near-zero metamaterials with gain media, Appl. Phys. Lett. 103 (2013) 201109.

[6] T. G. Mackay, A. Lakhtakia, Dynamically controllable anisotropic metamaterials with simultaneous attenuation and amplification, Phys. Rev. A 92 (2015) 053847.

[7] A. Cerjan, A. Fan, Achieving arbitrary control over pairs of polarization states using complex birefringent metamaterials, Phys. Rev. Lett. 118 (2017) 253902.

[8] T. G. Mackay, A. Lakhtakia, Simultaneous amplification and attenuation in isotropic chiral materials, J. Opt. (UK) 18 (2016) 055104.

[9] T. G. Mackay, A. Lakhtakia, Polarization-state-dependent attenuation and amplification in a columnar thin film, J. Opt. (UK) 19 (2017) 12LT01. Erratum 20 (2018) 019501.

[10] F. N. Marchevskii, V. L. Strizhevskii, S. V. Strizhevskii, Singular electromagnetic waves in bounded anisotropic media, Sov. Phys. Solid State 26 (1984) 911.

[11] M. I. D'yakonov, New type of electromagnetic wave propagating at an interface, Sov. Phys. JETP 67 (1988) 714.

[12] D. B. Walker, E. N. Glytsis, T. K. Gaylord, Surface mode at isotropic-uniaxial and isotropic-biaxial interfaces, J. Opt. Soc. Am. A 15 (1998) 248.

[13] O. Takayama, L.-C. Crasovan, S.K. Johansen, D. Mihalache, D. Artigas, L. Torner, Dyakonov surface waves: A review, Electromagnetics 28 (2008), 126.

[14] O. Takayama, L. Crasovan, D. Artigas, L. Torner, Observation of Dyakonov surface waves, Phys. Rev. Lett. 102 (2009) 043903.

[15] O. Takayama, D. Artigas, L. Torner, Practical dyakonons, Opt. Lett. 37 (2012) 4311.

[16] M. A. Noginov, Steering Dyakonov-like waves, Nat. Nanotechnol. 9 (2014) 414.

[17] S. Vuković, J. J. Miret, C. J. Zapata-Rodríguez, Z. Jakšić, Oblique surface waves at an interface between a metal-dielectric superlattice and an isotropic dielectric, Phys. Scripta 149 (2012) 014041. 
[18] Y.-L. Zhang, Q. Zhang, X.-Z. Wang, Extraordinary surface polaritons in obliquely truncated dielectric/metal metamaterials, J. Opt. Soc. Am. B 33 (2016) 543.

[19] A. Lakhtakia, J. A. Polo, Jr., Dyakonov-Tamm wave at the planar interface of a chiral sculptured thin film and an isotropic dielectric material, J. Eur. Opt. Soc.-Rapid Pub. 2 (2007) 07021.

[20] T. G. Mackay, A. Lakhtakia, Temperature-mediated transition from Dyakonov surface waves to surfaceplasmon-polariton waves, IEEE Photonics J. 8 (2016) 4802813.

[21] A. N. Furs, V. M. Galynsky, L. M. Barkovsky, Surface polaritons in symmetry planes of biaxial crystals, J. Phys. A: Math. Gen. 38 (2005) 8083.

[22] J. A. Polo Jr., T. G. Mackay, A. Lakhtakia, Electromagnetic Surface Waves: A Modern Perspective (Elsevier, 2013), Chap. 4.

[23] H. C. Chen, Theory of Electromagnetic Waves: A Coordinate-Free Approach (McGraw-Hill, 1983), Chap. 1.

[24] M. Born, E. Wolf, Principles of Optics, 7th (expanded) Edn. (Cambridge Univ. Press, 1999), Chap. 15.

[25] T. G. Mackay, A. Lakhtakia, Nonreciprocal Dyakonov-wave propagation supported by topological insulators, J. Opt. Soc. Amer. B 33 (2016) 1266.

[26] W. S. Weiglhofer, A. Lakhtakia, J. C. Monzon, Maxwell-Garnett model for composites of electrically small uniaxial objects," Microwave Opt. Technol. Lett. 6 (1993) 681.

[27] T. G. Mackay, Towards metamaterials with giant dielectric anisotropy via homogenization: an analytical study, Photon. Nanostruct. - Fund. Applicat. 13 (2015) 8. 